\title{
Evolutionary history and the strength of species interactions: testing the phylogenetic limiting similarity hypothesis
}

\author{
Keith J. Fritschie, ${ }^{1,3}$ Bradley J. Cardinale, ${ }^{1}$ Markos A. Alexandrou, ${ }^{2}$ and Todd H. Oakley ${ }^{2}$ \\ ${ }^{1}$ School of Natural Resources and Environment, University of Michigan, Ann Arbor, Michigan 48109 USA \\ ${ }^{2}$ Ecology, Evolution, and Marine Biology, University of California, Santa Barbara, California 93106 USA
}

\begin{abstract}
A longstanding concept in community ecology is that closely related species compete more strongly than distant relatives. Ecologists have invoked this "limiting similarity hypothesis" to explain patterns in the structure and function of biological communities and to inform conservation, restoration, and invasive-species management. However, few studies have empirically tested the validity of the limiting similarity hypothesis. Here we report the results of a laboratory microcosm experiment in which we used a model system of 23 common, co-occurring North American freshwater green algae to quantify the strength of 216 pairwise species' interactions (the difference in population density when grown alone vs. in the presence of another species) along a manipulated gradient of evolutionary relatedness (phylogenetic distance, as the sum of branch lengths separating species on a molecular phylogeny). Interspecific interactions varied widely in these bicultures of phytoplankton, ranging from strong competition (ratio of relative yield in polyculture vs. monoculture $\ll 1$ ) to moderate facilitation (relative yield $>1$ ). Yet, we found no evidence that the strength of species' interactions was influenced by their evolutionary relatedness. There was no relationship between phylogenetic distance and the average, minimum (inferior competitor), nor maximum (superior competitor) interaction strength across all biculture communities (respectively, $P=$ $0.19, P=0.17, P=0.14 ; N=428$ ). When we examined each individual species, only $17 \%$ of individual species' interactions strengths varied as a function of phylogenetic distance, and none of these relationships remained significant after Bonferoni correction for multiple tests $(N=23)$. Last, when we grouped interactions into five qualitatively different types, the frequency of these types was not related to phylogenetic distance among species pairs $\left(F_{4,422}=\right.$ $1.63, P=0.15)$. Our empirical study adds to several others that suggest the biological underpinnings of competition may not be evolutionarily conserved, and thus, ecologists may need to re-evaluate the previously assumed generality of the limiting similarity hypothesis.
\end{abstract}

Key words: coexistence; competition; competition-relatedness hypothesis; limiting similarity; phylogenetic diversity; phytoplankton.

\section{INTRODUCTION}

A widely held tenet of community ecology is that closely related species compete more strongly than distant relatives (Cahill et al. 2008). This tenet dates back to Darwin (1859), who interpreted the tendency of distantly related plants to be the most successful invaders as evidence that competition must, for whatever reason, be strongest among congeners (Daehler 2001). The idea that closely related taxa compete most strongly was formalized almost a century later as the limiting similarity hypothesis (MacArthur and Levins 1967); a hypothesis that is now a fundamental assumption of ecological theories on competition and coexistence, community assembly (Weiher and Keddy 1995), and biodiversity impacts on ecosystem functioning

Manuscript received 25 May 2013; revised 10 October 2013; accepted 15 October 2013. Corresponding Editor: S. Findlay.

${ }^{3}$ Present address: School of Aquatic and Fishery Sciences, University of Washington, Seattle, Washington 98105 USA. E-mail:kfritsch@uw.edu
(Cadotte et al. 2008, Connolly et al. 2011, Flynn et al. 2011, Srivastava et al. 2012).

Although the concept of limiting similarity is routinely invoked in community ecology, and increasingly so in applied fields like invasion (Catford et al. 2009) and restoration ecology (Verdú et al. 2012), it has been subjected to surprisingly little empirical validation. Manipulative experiments testing limiting similarity require logistically demanding designs with even moderately sized species pools (Cahill et al. 2008). Moreover, phylogenetic trees that have historically been based on taxonomy alone provide only a qualitative, noncontinuous metric of species relatedness.

The advent of molecular tools for describing species relatedness has renewed interest in testing the limiting similarity hypothesis (Cavender-Bares et al. 2009). The modern phylogenetic limiting similarity hypothesis (Violle et al. 2011) uses evolutionary history, as quantified by molecular phylogenies (e.g., Faith 1992, Ives and Helmus 2010), to describe evolutionary relatedness among species. In doing so, this approach 
implicitly assumes that the ecological traits that influence competition are conserved along a phylogenetic lineage such that closely related species tend to occupy similar niches (Wiens and Graham 2005). Thus, according to phylogenetic limiting similarity, species more closely related in evolutionary time are more ecologically similar and compete more strongly than distant relatives.

Although patterns in the phylogenetic structure of natural communities are often used to support the limiting similarity hypothesis (Webb et al. 2002), recent theoretical studies suggest that multiple biological (e.g., niche differences, fitness differences, environmental filtering; Mayfield and Levine 2010) and nonbiological (random Brownian evolution; Losos 2008) processes can interact to determine a community's phylogenetic structure. Thus, the phylogenetic structure of natural communities (a pattern) should not be considered an explicit test of limiting similarity (a biological process). Controlled manipulative experiments offer a better test of the phylogenetic limiting similarity hypothesis by permitting the explicit measurement of interaction strengths in the absence of confounding factors. Cahill et al. (2008) represents one of the first to attempt this approach, integrating a molecular phylogeny with a meta-analysis of competition experiments to show that there was little evidence of limiting similarity in terrestrial plant communities. Recent experimental studies (Jiang et al. 2010, Burns and Strauss 2011, Violle et al. 2011, Peay et al. 2012, Tan et al. 2012, Best et al. 2013) have reported contrasting relationships between evolutionary relatedness and the strength of competitive interactions. Given the logistic constraints of multispecies interaction experiments, these studies can only accommodate tens of focal communities $(N=15$, Jiang et al. 2010; $N=36$, Burns and Strauss 2011; $N=45$, Violle et al. 2011; $N=30$, Peay et al. 2012; $N=20$, Tan et al. 2012; $N=18$, Best et al. 2013), which may yield results that are highly contingent on the phylogenetic scale of inquiry (e.g., Peay et al. 2012: Fig. 4). Additionally, many are not designed to explicitly test the phylogenetic limiting similarity hypothesis (i.e., to relate variation in interspecific interaction strengths to the evolutionary divergence between the interacting species), but rather to search for a signal of limiting similarity while testing more complex phylogenetic community ecology principles (e.g., priority effects, ecosystem functioning, invasibility; but see Violle et al. 2011 and Burns and Strauss 2011).

Here we add to this growing body of literature by providing a straightforward test of phylogenetic limiting similarity using a large species pool distributed across a variety of phylogenetic distances. Our experiment draws on 23 species from 17 genera of naturally co-occurring freshwater green algae described by a new molecular phylogeny. We cultured each species alone and in all pairwise combinations in $1-\mathrm{mL}$ well plate microcosms and, after allowing 10-40 generations of population dynamics in a stable environment, measured the interspecific interaction strengths of 864 populations in 216 replicated biculture communities. We found no evidence for phylogenetic limiting similarity at several scales of phylogenetic inference, ranging from speciesspecific to whole-species pool analyses. Echoing recent ecological and evolutionary theory, we speculate as to why phylogenetic limiting similarity may not be pervasive in ecological systems, while noting the limitations of our own study.

\section{Methods \\ Phylogeny}

A phylogeny was constructed that included 37 common North American freshwater green algae genera using partial $18 \mathrm{~S}$ ribosomal RNA and rbcl sequences that were available on GenBank. We targeted these molecular markers as they provided the most complete sets of data for the target species used in the experiments. We extended taxon sampling outside the target species to include representative taxa from the Chlorophyta and Charophyta, thereby placing our experimental species pool within a broader phylogenetic framework. By increasing taxonomic sampling we were also able to assess the taxonomic stability of the experimental species under investigation (i.e., if the molecular phylogeny recovered different taxonomic hierarchies as monophyletic groups). Three species were used as outgroups based on previous phylogenetic results (Rodriguez-Ezpeleta et al. 2005). We constructed alignments independently for each gene using MUSCLE v 3.8.31 (Edgar 2004). Best-fit nucleotide substitution models were selected for each gene using the Akaike information criterion as implemented in jModelTest $\mathrm{v}$ 0.1.1 (Posada 2008). An unsmoothed maximum likelihood phylogeny was constructed using RAxML v 7.2.8 (Stamatakis et al. 2008). The analysis was partitioned by gene using a mixed partition model, random starting trees used for each independent tree search and topological robustness was investigated using 100 nonparametric bootstrap replicates. We refer to branch lengths as "unsmoothed" when they represent the average number of mutational changes per site present in the alignment. In addition, we constructed "smoothed" branch lengths by implementing a relaxed molecular clock, which better represent estimates of time since common ancestry by forcing the tree topology to be ultrametric. For smoothed branch lengths, we constructed a Bayesian phylogeny using BEAST v1.6.2 (Drummond and Rambaut 2007), assuming a relaxed uncorrelated lognormal clock and all other parameters on default. The Bayesian analysis ran for 10 million generations sampled every 1000 generations, while stationarity and effective sample sizes (ESS $>200)$ were examined using Tracer v1.5 (software available online), ${ }^{4}$

${ }^{4}$ http://beast.bio.ed.ac.uk/Tracer 
discarding all trees under the asymptote. We constructed a consensus tree with mean node heights from the posterior distribution using Tree Annotator v1.6.2 (Appendix A: Fig. A1; Drummond and Rambaut 2007).

The evolutionary relatedness of community members is inversely proportional to the community's phylogenetic diversity (PD). A growing number of metrics have been developed to describe the PD of communities (e.g., Webb et al. 2002, Pavoine et al. 2005, Helmus et al. 2007), with most intended to account for two confounding factors in traditional PD metrics: species richness and abundance (Cadotte et al. 2010).

In our experiment, we held richness constant $(N=2)$, and we explicitly used relative density calculations to account for variation in the final abundances of species so that we could ask how initial, manipulated evolutionary divergence between community members influences final relative densities. Since our experimental design already controls for richness, and because change in relative abundance is our response variable, we have foregone new PD metrics and used Faith's (1992) more straightforward measure of the phylogenetic distance between two species. We calculated PD using a custom Bioperl script (Stajich et al. 2002) from mean branch lengths connecting each species pair, ignoring the root branch. In several cases, sequences were not available for our experimental species (Ankistrodesmus falcatus, Coelastrum cambricum, Oocystis polymorpha, Planktosphaeria botryoides). We therefore used distances for the genus rather than the species, or in the case of $C$. cambricum, the average of the distances between the competitor and each remaining species of the Coelastrum genus (C. reticulatum and C. microporum).

\section{Experiment}

Experimental communities were assembled from 23 green algae species chosen from our new phylogeny (Appendix B: Table B1). Species were chosen hierarchically according to their ecological relevance, their availability in culture, their ability to grow in a common culture medium (COMBO; Kilham et al. 1998), and their visual distinguishability via compound microscopy. According to the U.S. Environmental Protection Agency's National Lakes Assessment (U.S. EPA $2009)$, the 23 chosen species occurred in $3 \%$ to $71 \%$ of the 1100 lakes sampled, representing the 2nd (Oocystis spp.) to 110th (Chlamydocapsa spp.) most common phytoplankton genera out of 262 reported. Their ecological distributions suggest that the species used in this experiment do co-occur in temperate North American lakes, and thus serve as a relevant model system to test the limiting similarity hypothesis (see Plate 1). Cultures of the focal species were originally obtained from Sammlung von Algenkulturen Gottingen (Gottingen, Germany), the Culture Collection of Algae and Protozoa (Oban, UK), the Canadian Phycological Culture Centre (Ontario, Canada), and the UTEX Culture Collection of Algae (Austin, Texas, USA).
Batch cultures of each species were grown under experimental conditions for two weeks prior to the experiment.

The 23 species were grown in replicate monoculture and in all pairwise biculture combinations. Using a replacement-series (i.e., substitutive) design, species were inoculated at 5000 cells $/ \mathrm{mL}$ each within a biculture and 10000 cells $/ \mathrm{mL}$ within a monoculture, which is, on average, $<1 \%$ of species' final monoculture densities $(\mathrm{SD}=2.98$, maximum $=14.5 \%)$. Each of the 23 monocultures and 253 bicultures was replicated twice, resulting in 552 total species/species assemblages.

Species/species assemblages were cultured in clear polystyrene 48-well plates (number 677102, Greiner Bio One, Monroe, North Carolina, USA) containing $1 \mathrm{~mL}$ of standardized COMBO growth medium. Each plate contained both replicates of six pairwise combinations of species. Combinations were assigned to plates nonrandomly ordered by species identification (ID) number (Appendix B: Table B1), but plates were then randomized across three shaker tables (MaxQ 2000; Thermo Fisher Scientific, Fife, Washington, USA) (See Appendix C: Fig. C1 for illustrated experimental design.) This design was preferred over a completely randomized assignment to well-plates to minimize (1) time differences between species inoculations within and across communities (total inoculation period $\sim 10$ hours), (2) the potential for cross-contamination by containing motile species to as few plates as possible, and (3) technician error during inoculation, medium exchanges, and sampling by providing an intuitive way to keep track of a large number $(N=552)$ of microbial assemblages. At the same time, this design ensured that the focal independent variable (evolutionary relatedness) was distributed randomly across the experimental units. Shaker tables were set up in a climate-controlled environmental room (Enviro-line; Nor-lake, Hudson, Wisconsin, USA) where they were held at $18.0^{\circ} \mathrm{C}$, continuously rotated at $0.4 \mathrm{~m} / \mathrm{s}$ (115 RPM), and exposed to $4100 \mathrm{~K}$ fluorescent bulbs operating on a $16: 8$ hour light: dark cycle that emitted $120 \pm 5 \mu \mathrm{mol}$ photons $\cdot \mathrm{m}^{-2} \cdot \mathrm{s}^{-1}$. Medium was exchanged by manual pipetting at a rate of $20 \%$ every other day to replenish essential nutrients.

In vivo fluorescence of chlorophyll $a$ (excitation $=460$ $\mathrm{nm}$, emission $=685 \mathrm{~nm}$ ) was measured every other day using a multimode plate reader (number H1M; Biotek, Winooski, Vermont, USA) and fluorescence time series were used to track algal growth dynamics. After 40 days, the experiment was terminated when the majority of cultures no longer exhibited positive growth (see Appendix D: Fig. D1 for monoculture growth curves). While total biomass in cultures appeared to reach a steady state, we cannot say whether the populations of each individual species in a biculture were stable by the end of the experiment, as documenting steady state for each population would have required counting a prohibitive number of samples (10 time points $\times 552$ 
replicates $=5520$ density counts). However, we can say that the 40-day duration of the experiment was sufficient for 10-40 generations of algal growth (depending on species), which is considerably longer than the duration of the vast majority of past competition studies (Ives et al. 2003).

At the end of the experiment, $600 \mu \mathrm{L}$ of each replicate were preserved in $4 \%$ formalin. Species' densities were enumerated using a hemacytometer and compound microscope at $400 \times$ magnification, counting at least 400 cells per sample. Thirty-seven biculture treatments were removed from analyses because morphological distortion from formalin preservation rendered them indistinguishable (mean phylogenetic distance of study and removed populations were not significantly different [study PD mean $=0.176$, removed PD mean $=0.171, P=$ $0.79]$ ), and an additional four replicates were removed because they were invaded by species from neighboring treatments. Thus, the final data set included 23 species, 216 biculture treatments, and 428 replicate bicultures.

\section{Interaction metrics and analysis}

Ecologists have used many different metrics to quantify the strength of species interactions (Laska and Wootton 1998) and competitive interactions among plants specifically (Weigelt and Jolliffe 2003). The use of relative yield (RY) as a measure of interaction strength (the ratio of a species' yield when grown in the presence of others and its yield when grown in monoculture) was first proposed by de Wit (1960) and applied by modern agronomists interested in how intercropping impacts biomass yield (Harper 1977, Vandermeer 1989). Ecologists have since used RY in studies of species interactions and biodiversity effects on ecosystem functioning (Loreau and Hector 2001). In the same vein as relative yields, we used algal species' relative densities (RD) to quantify interaction strengths. The relative density of each species $i, \mathrm{RD}_{i}$, was calculated as the ratio of species $i$ 's population density in biculture: monoculture ratio. This measure assumes that cell biovolumes are fixed, and thus, species interactions are manifest primarily through changes in population size as opposed to variation in the growth or size of individual cells.

Some have expressed concerns about the interpretability of relative yield/density measures for replacement-series experimental designs (Austin et al. 1988, Snaydon 1991, Jolliffe 2000). These concerns are directed at classic plant interaction studies that compare a species' vegetative yield when grown at some fixed monoculture density to its yield when grown at a different fixed biculture density. Under these conditions, the replacement-series relative yield can be influenced by both interspecific interactions and intraspecific differences in density-dependent vegetative growth rates. Note, however, that this criticism is not relevant to our study, since initial species densities were not held constant, but instead were allowed to undergo long-term population dynamics before we compared monoculture and biculture treatments. Thus, the final population sizes of algae measured in our study were the product of any and all forms of interaction occurring in the cultures, including both intra- and interspecific.

$\mathrm{RD}$ is a property of individual species. Each species potentially has a unique value of RD, and any two species grown in biculture may have very different values of RD due to asymmetry in interaction strengths. In contrast, $\mathrm{PD}$ is a property of the community and, in our study, each biculture has just one value. The mismatch between a species-level and community-level property required that we examine the data using a sequence of analyses that test for potential differences in interaction strength and symmetry along a PD gradient. First, we regressed the average biculture RD, the inferior species RD (i.e., lowest RD), and the superior species RD (i.e., highest RD) on the phylogenetic distance between species. The null hypothesis is that each of these relationships should be positive, indicating that competitive interactions weaken as the relatedness among species declines. Second, we regressed the variance and absolute difference of RD's among interacting species' $\left|\mathrm{RD}_{i}-\mathrm{RD}_{j}\right|$ on phylogenetic distance to assess whether interactions grow more asymmetric with PD.

Third, we plotted species' RDs simultaneously in bivariate space to relate six qualitative forms of interspecific interactions to evolutionary relatedness. The null expectation for relative yield/density analyses is that inter- and intraspecific interactions are equal among species. Under this assumption, any species initially inoculated in a 50:50 proportion in biculture should reach exactly half of its monoculture density as if it were interacting with a distinguishable population of the same species $(\mathrm{RD}=0.5)$. If instead a species experiences no interspecific interactions in biculture, it should be limited only by intraspecific competition, and thus, reach a density that is equal to what it achieves in monoculture $(\mathrm{RD}=1.0)$. Alternatively, if a species is facilitated by the presence of another, it will reach a greater density than what it achieves when grown alone $(\mathrm{RD}>1.0)$. If interspecific competition occurs, but is weaker than intraspecific competition (a necessary condition for stable coexistence, Chesson 2000), then a species should reach densities that are lower than its monoculture value, but greater than its density when inter- and intraspecific interactions are equal $(0.5<\mathrm{RD}$ $<1.0)$. Conversely, if interspecific competition is stronger than intraspecific competition (indicative of unstable coexistence) a species should achieve a lower density than when inter- and intraspecific competition are equal $(\mathrm{RD}<0.5)$.

\section{RESUlts}

We found strong evidence of competitive interactions among green algal species. On average, species' population sizes (cell densities) were reduced by $41 \%$ when grown in the presence of a second species compared to their monoculture population sizes (mean $\mathrm{RD}=0.60$, 

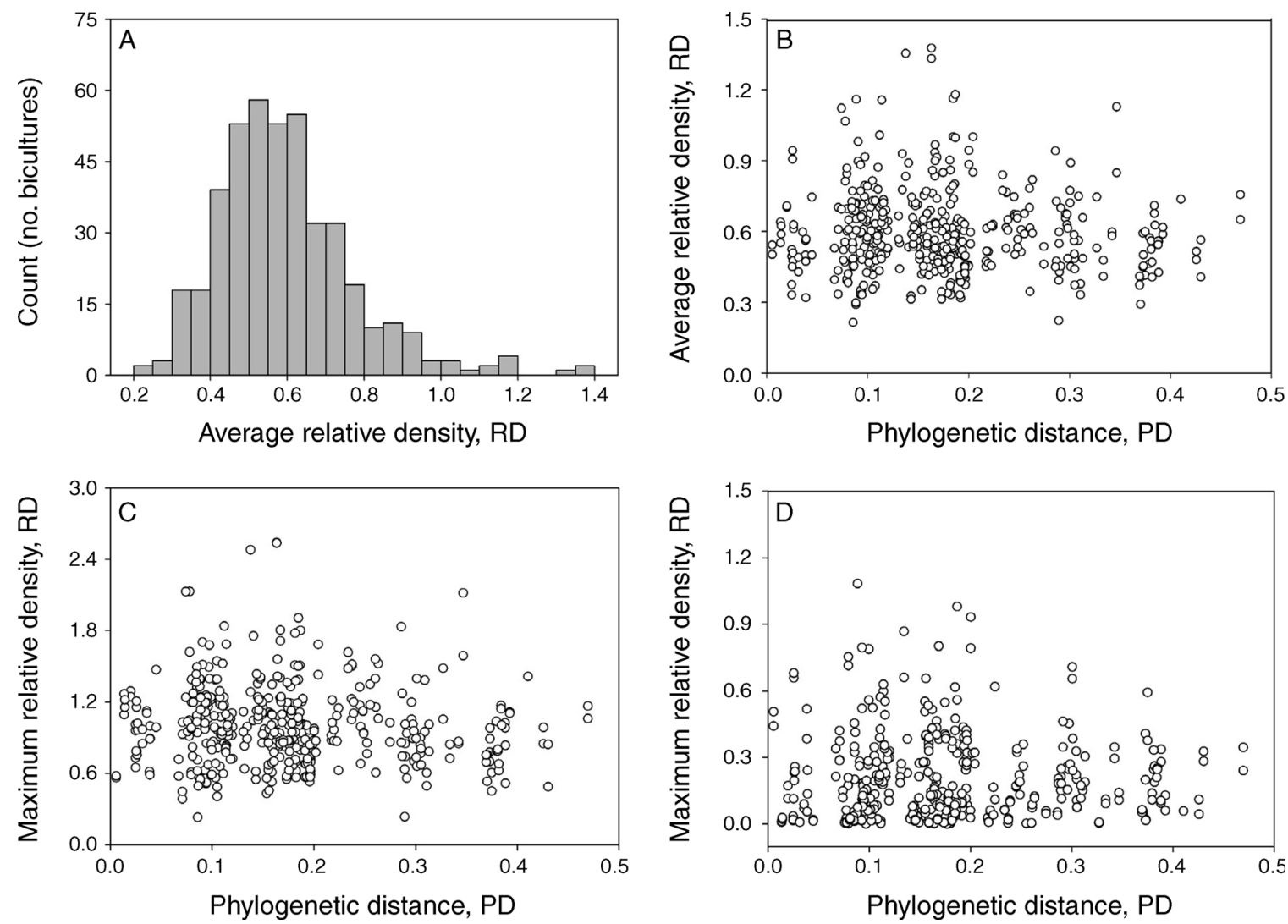

FIG. 1. Impacts of evolutionary relatedness on the strength of interspecific competition. (A) The distribution of species' relative densities in 428 experimental phytoplankton bicultures. Densities were reduced by a mean $41 \%$ relative to their monoculture value when they were grown in the presence of another species (average relative density $[R D]=0.60, S D=0.18$ ). This result indicates a prevalence of interspecific competition in species bicultures, but note that responses ranged from strong competition (RD $\ll 1)$ to moderate facilitation (RD > 1). However, (B) the strength of interspecific competition, as measured by the size of the average RD of a biculture, was not significantly related to the phylogenetic distance between species. The RDs of the superior (C) and inferior (D) competitors also showed no significant relationship to their phylogenetic distance. Data in panel (D) were logit-transformed for analysis to normalize a right-skewed distribution, but are plotted without transformation to facilitate comparison with the other panels.

$\mathrm{SD}=0.18, N=428$; Fig. 1A). It is noteworthy, however, that interspecific interactions spanned a large gradient ranging from very strong competition (RD's $\rightarrow 0$ ) to moderate facilitation $(\mathrm{RD}>1)$. Despite large variation in the nature of interspecific interactions, the average interaction strength did not vary as a function of the evolutionary relatedness separating interacting species (mean $\mathrm{RD}=0.62-0.12 \times \mathrm{PD}, r^{2}<0.01, P=0.19, N=$ 428; Fig. 1B). Parsing the average interaction strength into those most vs. least influenced by the presence of the other taxa led to equivalent results; there was no relationship between phylogenetic distance and the RDs of the least (superior species $\mathrm{RD}=1.04-0.25 \times \mathrm{PD}, r^{2}$ $<0.01, P=0.14, N=428$; Fig. $1 \mathrm{C}$ ) or most (inferior species $\mathrm{RD}=-2.28+1.45 \times \mathrm{PD}, r^{2}<0.01, P=0.17, N=$ 427; logit-transformed to normalize a right-skewed distribution; Fig. 1D) impacted species. We also found that competitive asymmetries, as measured by both the variance and absolute difference of species' RDs in biculture, did not vary with phylogenetic distance (variance in $\mathrm{RD}=0.47-0.31 \times \mathrm{PD}, r^{2}<0.01, P=$ $0.12, N=428$; absolute difference in $\mathrm{RD}=0.85-0.29 \times$ PD, $\left.r^{2}<0.01, P=0.20, N=428\right)$. Thus, when data were lumped to consider 428 combinations of phytoplankton species, we found no evidence to support the limiting similarity hypothesis that distantly related algal species experience weaker interspecific competition than closely related species.

We analyzed the data set in more detail to look for evidence of phylogenetic limiting similarity within each individual species. To do so, we regressed each of the 28 focal species' RD on the PD between it and its competitors. Using liberal statistical tests (no Bonferoni correction for multiple comparisons), we found that $17 \%$ of the species pool exhibited a significant, albeit weak, negative relationship between RD and PD (Golenkinia minutissima, $b=-6.01, r^{2}=0.34, P<0.01, N=23$; Oocystis polymorpha, $b=-3.56, r^{2}=0.19, P<0.01, N=$ 38; Planktosphaeria botryoides, $b=-5.90, r^{2}=0.17, P=$ $0.03, N=26$; Selenastrum capricornutum, $b=-1.51, r^{2}=$ 
TABLE 1. Summary of the effect of phylogenetic distance between a focal species and its competitor (PD) on the relative density (RD) of the focal species: $\mathrm{RD}=a+b(\mathrm{PD})$

\begin{tabular}{|c|c|c|c|c|c|}
\hline Species & $N$ & $a(\mathrm{SE})$ & $b(\mathrm{SE})$ & $R^{2}$ & $P$ \\
\hline Ankistrodesmus falcatus & 41 & $0.333(0.11)$ & $-0.391(0.70)$ & 0.008 & 0.580 \\
\hline Botryococcus sudeticus & 24 & $1.669(0.450)$ & $-2.472(1.777)$ & 0.078 & 0.178 \\
\hline Chlamydocapsa ampla & 34 & $0.953(0.154)$ & $-0.401(0.68)$ & 0.010 & 0.561 \\
\hline Chlamydomonas moewusii & 33 & $1.177(0.275)$ & $-1.924(1.218)$ & 0.072 & 0.124 \\
\hline Chlorella sorokiniana & 29 & $0.644(0.259)$ & $-1.740(1.349)$ & 0.056 & 0.208 \\
\hline Closteriopsis acicularis & 43 & $1.210(0.397)$ & $-3.365(2.120)$ & 0.057 & 0.120 \\
\hline Coelastrum cambricum & 33 & $0.532(0.119)$ & $1.364(0.738)$ & 0.096 & 0.074 \\
\hline Coelastrum microporum & 33 & $0.145(0.046)$ & $-0.001(0.314)$ & $>0.001$ & 0.996 \\
\hline Coealstrum reticulatum & 31 & $0.794(0.163)$ & $0.106(0.964)$ & $>0.001$ & 0.914 \\
\hline Cosmarium botrytis & 43 & $0.741(0.596)$ & $-0.032(1.934)$ & $>0.001$ & 0.987 \\
\hline Gloecystis gigas & 33 & $0.852(0.165)$ & $0.841(0.861)$ & 0.029 & 0.336 \\
\hline Golenkinia minutissima & 23 & $1.535(0.344)$ & $-6.006(1.784)$ & 0.340 & 0.003 \\
\hline Monoraphidium arcuatum & 41 & $0.965(0.150$ & $-1.616(0.907)$ & 0.073 & 0.082 \\
\hline Monoraphidium minutum & 41 & $0.796(0.123)$ & $-0.688(0.729)$ & 0.022 & 0.351 \\
\hline Oocystis polymorpha & 38 & $1.819(0.468)$ & $-3.558(1.228)$ & 0.185 & 0.006 \\
\hline Pediastrum boryanum & 43 & $0.965(0.083)$ & $-0.643(0.490)$ & 0.039 & 0.196 \\
\hline Pediastrum duplex & 41 & $0.446(0.099)$ & $0.131(0.595)$ & 0.001 & 0.826 \\
\hline Pediastrum tetras & 40 & $0.099(0.059)$ & $0.078(0.363)$ & 0.001 & 0.831 \\
\hline Plantosphaeria botryoides & 26 & $1.459(0.323)$ & $-5.903(2.574)$ & 0.174 & 0.030 \\
\hline Scenedesmus acuminatus & 43 & $0.831(0.097)$ & $-0.255(0.588)$ & 0.004 & 0.667 \\
\hline Scenedesmus obliquus & 40 & $1.009(0.099)$ & $-0.941(0.598)$ & 0.060 & 0.124 \\
\hline Selenastrum capricornutum & 40 & $0.509(0.127)$ & $-1.506(0.676)$ & 0.113 & 0.032 \\
\hline Tetraedron minutum & 41 & $0.225(0.089)$ & $-0.603(0.566)$ & 0.028 & 0.294 \\
\hline
\end{tabular}

$0.11, P=0.03, N=40$; Table 1$)$. When test statistics were corrected for multiple comparisons, none of these relationships remained significant (Bonferroni corrected $P=\alpha / N=0.05 / 23=0.0022)$. Thus, both liberal and conservative statistical tests provided only weak support or no support at all, respectively, for phylogenetic limiting similarity.

We further subdivided species' RDs into categories that give more detail about the nature of the species interactions, possible competitive asymmetries, and their potential relationship with evolutionary relatedness. Each species in a biculture may experience either strong competition (stronger inter- than intraspecific competition, RD $<0.5$ ), weak competition (stronger intra- than interspecific competition, $1>\mathrm{RD}>0.5$ ), or facilitation $(\mathrm{RD}>1.0)$. We plotted the joint distribution of species' RDs for each biculture to examine how many interactions fell within each of these categories, and to examine potential asymmetries in interaction strengths (Fig. 2). Interaction ranged from (1) mutually strong competition (both species' RDs $<0.5, N=12$ ) to (2) mutual facilitation (both species' RDs $>1.0, N=1$ ). However, the vast majority of interactions fell within intermediate scenarios in which (3) both species experienced weak interspecific competition (1.0 $\left.>\mathrm{RD}_{x}>0.5 ; N=23\right)$, (4) one species was facilitated while the other experienced weak interspecific competition $\left(\mathrm{RD}_{i}>1.0,1.0>\mathrm{RD}_{j}>\right.$ $0.5 ; N=10)$, (5) one species was facilitated while the other experienced strong competition $\left(\mathrm{RD}_{i}>1.0, \mathrm{RD}_{j}\right.$ $<0.5 ; N=186$ ), or 6 ) one species experienced weak competition while the other experienced strong competition $\left(1.0>\mathrm{RD}_{i}>0.5, \mathrm{RD}_{j}<0.5 ; N=196\right)$. Despite parsing species interactions into these finer gradations that also accounted for asymmetric interaction strengths, we found no evidence of a phylogenetic signal across the interaction gradient $\left(F_{4,422}=1.63, P=0.15\right.$; ANOVA between group results for interaction scenarios; note that mutual facilitation was removed because $N$ $=1)$.

Last, we evaluated whether there was any evidence that evolutionary relatedness influences the probability of coexistence among taxa. Coexistence, when strictly defined in theoretical models for stable, closed environments (Chesson 2000), only occurs when intraspecific competition is stronger than interspecific competition for both competitors in the biculture (i.e., $\mathrm{RD}_{x}>0.5$ ). According to the phylogenetic limiting similarity hypothesis, we would expect the likelihood of coexistence among competing taxa to increase as a function of phylogenetic distance. In contrast to this prediction, we found a weak, albeit significant, negative relationship between PD and the likelihood of coexistence $\left(\chi^{2}=4.26\right.$, $P=0.04, N=428$; Appendix E: Fig. E1). This negative result could be accounted for by two species, Cosmarium botrytis and Oocystis polymorpha, which were present in $67 \%$ of communities that had PD values $>0.2$. These two taxa generally experienced very strong competition ( RD $<0.25$ in $24.4 \%$ and $33.3 \%$ of combinations, respectively) or strongly outcompeted the other species (competitor RD $<0.25$ in $44.4 \%$ and $35.9 \%$ of combinations, respectively). After removing these two species from the analysis, there was no significant relationship between the likelihood of coexistence and phylogenetic distance $\left(O\right.$. polymorpha removed, $\chi^{2}=$ 2.55, $P=0.11, N=389 ; O$. polymorpha and $C$. botrytis removed, $\left.\chi^{2}=1.74, P=0.19, N=347\right)$. These results show that the probability of coexistence did not increase 


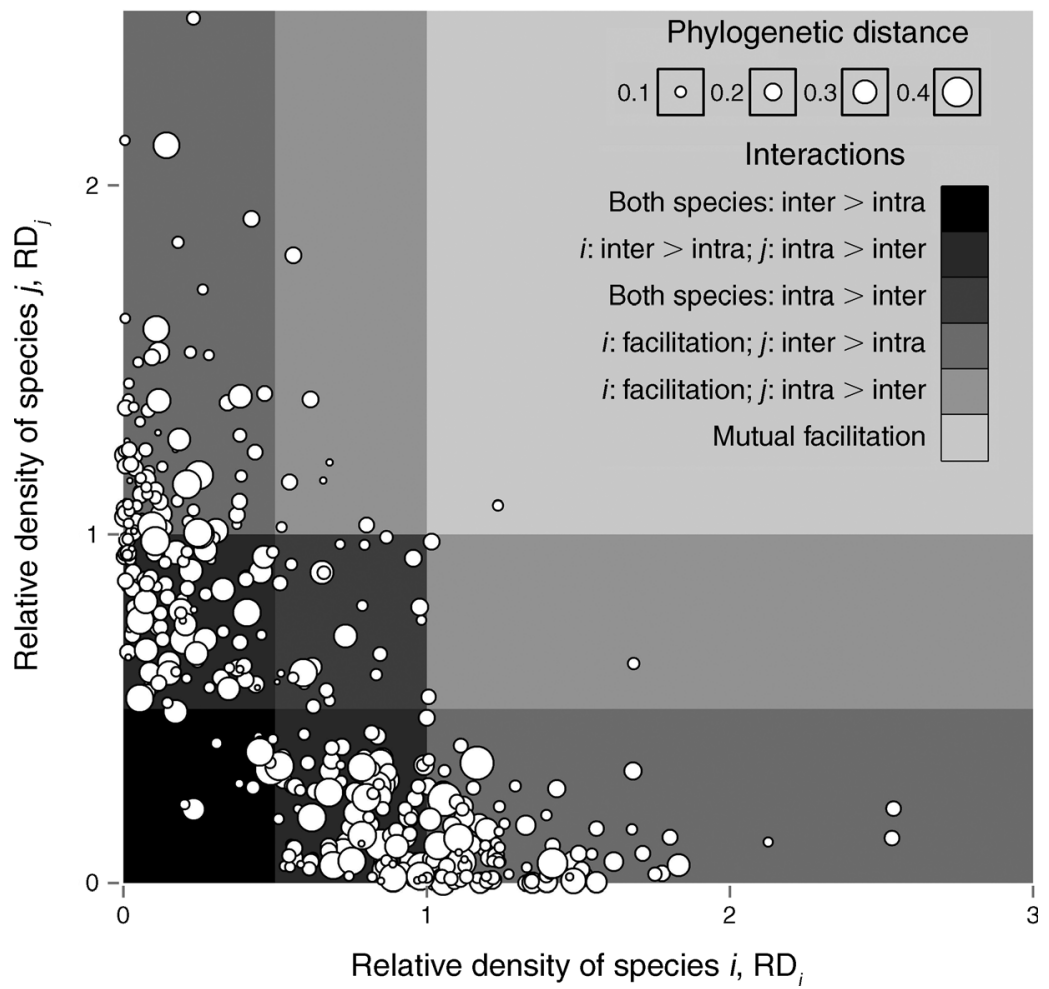

FIG. 2. The distribution of algae communities across an interaction gradient. We plotted the joint distribution of species' relative densities (each data point is a single biculture and its size is scaled to phylogenetic diversity [PD] between its constituent species) for a more detailed examination of species' interactions. The background color scales along a gradient of competition strength; the dark gray indicates that (1) both species experienced stronger interspecific (inter) vs. intraspecific (intra) competition $\left(\mathrm{RD}_{x}<0.5 ; N=12\right)$, while the lightest gray indicates that (2) species were mutually facilitated $\left(\mathrm{RD}_{x}>1 ; N=1\right)$. The majority of combinations fell in intermediate scenarios in which (3) both species experienced weak interspecific competition $\left(1>\mathrm{RD}_{x}>0.5 ; N\right.$ =23), (4) one species was facilitated while the other experienced weak interspecific competition $\left(\mathrm{RD}_{i}>1,1>\mathrm{RD}_{j}>0.5 ; N=10\right)$, (5) one species was facilitated while the other experienced strong competition $\left(\mathrm{RD}_{i}>1, \mathrm{RD}_{j}<0.5 ; N=186\right)$, and (6) one species experienced weak competition while the other experienced strong competition $\left(1>\mathrm{RD}_{i}>0.5, \mathrm{RD}_{j}<0.5 ; N=196\right)$. Despite this variation in species interactions, we found no evidence of a phylogenetic signal across this interaction gradient $\left(F_{4,422}=1.637, P=\right.$ 0.15; ANOVA between group results for the five interaction scenarios, with the mutual facilitation scenario removed because of sample size).

with evolutionary divergence among these green algal species.

\section{Discussion}

In an effort to explain patterns of community structure and function, ecologists frequently assume that closely related species compete more strongly than distant relatives. Despite this widespread assumption and its intuitive appeal, surprisingly few studies have actually tested the phylogenetic limiting similarity hypothesis. Of the few studies that have directly tested the hypothesis, inferences have been limited by the relatively small number of species combinations tested (which limits the phylogenetic range), and by the fact that many studies do not directly measure the strength of species interactions. We used a variety of analyses across 428 phytoplankton communities to examine how phylogenetic distance, as a measure of evolutionary relatedness, relates to species' relative densities, which is an explicit measure of the strength of interspecific interactions. Despite a large gradient in interaction strength, from strong competition to moderate facilitation, we found no evidence to support the hypothesis that phylogenetic distance influences the strength or symmetry of competitive interactions in this large and heterogeneous group of freshwater green algae.

Our results add to, and complement, a recent surge of studies that have attempted to determine how evolutionary history influences ecological interactions. A subset of these studies have, in fact, demonstrated a positive relationship between trait divergence, phylogenetic distance, and coexistence in protist communities (Violle et al. 2011), a negative relationship between priority effects and phylogenetic distance in yeast and bacterial communities (Peay et al. 2012, Tan et al. 2012), and a positive relationship between relative interaction intensity and phylogenetic distance in plant communities (Burns and Strauss 2011). But our results corroborate a different body of work that has found little influence of phylogenetic distance on competition and community 


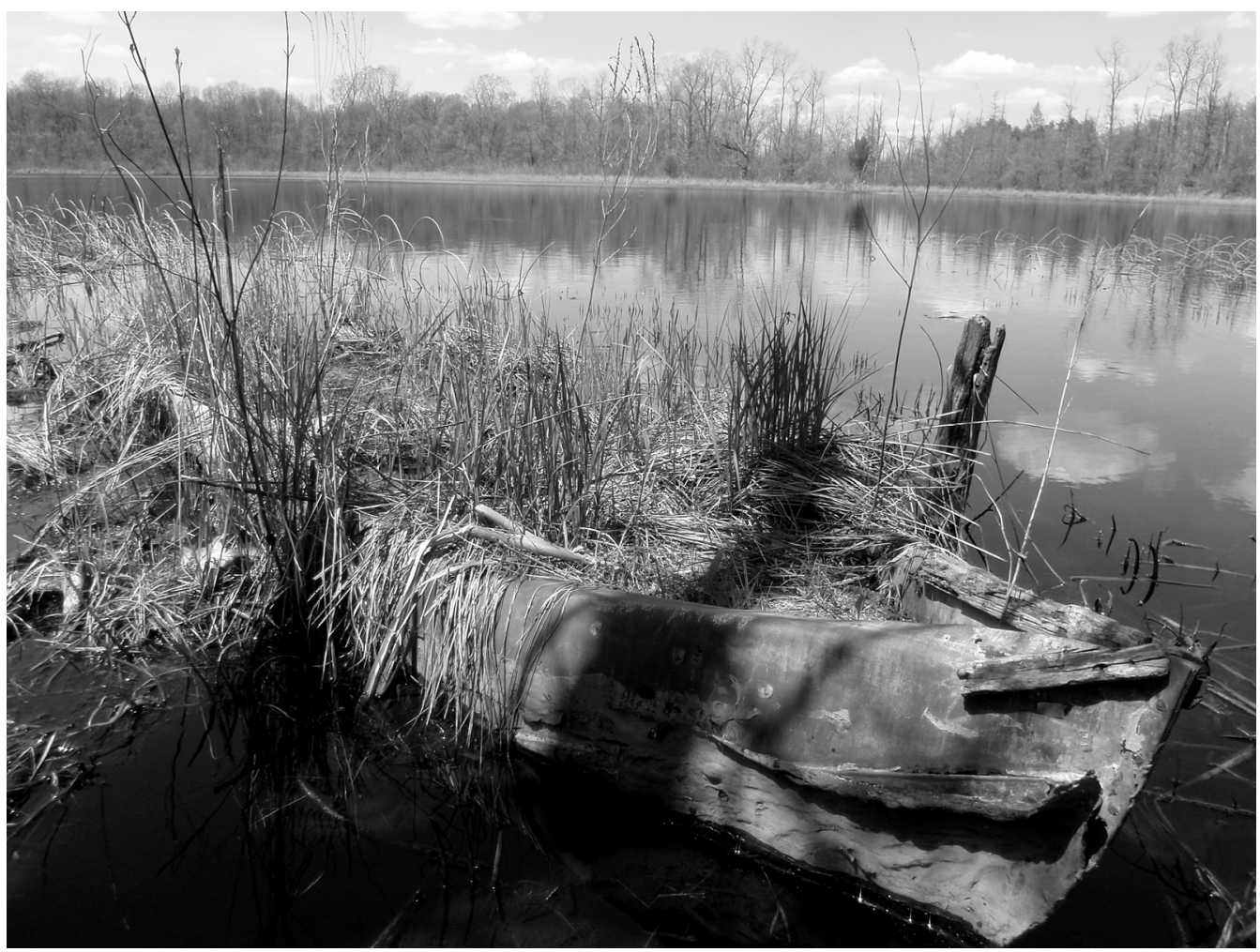

Plate 1. Species were selected based on their occurrence in the EPA's National Lakes Assessment data set. Sullivan Lake (pictured; Washtenaw County, Michigan, USA) was sampled as a reference site in the 2007 assessment. Photo credit: K. J. Fritschie.

assembly in plant, tree, and marine invertebrate systems (Cahill et al. 2008, Dostál 2011, Kunstler et al. 2012, Best et al. 2013). The mixed results and conclusions of studies to date may be attributable to alternative ecological and evolutionary mechanisms operating in different study systems, though it is perhaps too early to identify specific sources of variation in these varied data sets. Nevertheless, the mixed evidence and controversy suggest that biologists may want to be more conservative with claims about the pervasive influence of evolution on ecological interactions, at least until we can explain why half or more of existing case studies tend to show no phylogenetic signal.

Surprisingly, we did find that $23 \%$ of algal populations were facilitated in the presence of others, although that declined to $2.6 \%$ when considering only mutualistic $(+\quad+)$ and commensal $(+0)$, not antagonistic $(+-)$, interactions. Recent empirical work has found that the likelihood of facilitative interactions may increase along a gradient of species relatedness (Valiente-Banuet and Verdú 2007), but thus far this evidence has preceded explicit theory on PD facilitation relationships. Although not significant $\left(\chi^{2}=2.98, P=0.09, N=428\right)$, our analysis actually suggests the opposite trend; the most distantly related species pair had $e^{0.811}=2.25 \times$ lower odds of facilitative interactions than the most closely related pair. The conflicting results of these empirical studies indicate another area in which our understanding of evolutionary and ecological interactions needs to be strengthened.

\section{Potential ecological explanations: phylogenetic conservatism and trait trade-offs}

The phylogenetic limiting similarity hypothesis is an intuitive and appealing concept, but there are several reasons why phylogenetic signal may be absent from species' interactions. Although we cannot conclude which mechanisms contribute to the lack of signal in our own results, we discuss a few possibilities below for consideration in future studies that invoke the concept of limiting similarity.

Suites of morphological, physiological, behavioral, and life history traits ultimately govern species' abiotic and biotic interactions (see Litchman and Klausmeier 2008 for a review of traits that are relevant to phytoplankton ecology). The validity of limiting similarity requires that the traits governing species interactions exhibit phylogenetic signal (evolutionary divergence of trait values that exceed the magnitude produced by a random Brownian motion model) and phylogenetic conservatism (trait divergence that proceeds at a rate lower than expected by chance). Phylogenetic signal or conservatism may be induced through evolutionary mechanisms like gene flow, low 
genetic diversity, stabilizing selection, and diversification deep within a clade (Harvey and Pagel 1991, Wiens and Graham 2005, Crisp and Cook 2012). However, recent studies have found that phylogenetic signal and niche conservatism may be the exception rather than the rule in some clades and have opened a discussion on why these phylogenetic patterns may not exist for certain types of organisms (Losos 2008, Pearman et al. 2008; but see Wiens et al. 2010). Perhaps the most obvious potential cause is convergent trait evolution among distant relatives. If species within a clade diverge to adapt to local environmental conditions and this occurs across clades within a given region, we might expect distant relatives to be more ecologically similar than those within the same clade (Losos 2008). By mediating the relative fitness of species in a given environment, convergent evolution of environmental traits can certainly have implications for the strength of species interactions that do not follow from a direct examination of phylogenetic pattern (Cavender-Bares et al. 2006).

Furthermore, the impact of competitive interactions has the potential to decrease as species become more ecologically similar to one another. Contemporary coexistence theory suggests that species interactions are driven by a balance of competitors' niche differences (NDs are stabilizing mechanisms that promote positive growth rates when a species is rare) that weaken interspecific competition, and relative fitness differences (RFDs are equalizing mechanisms such as resource acquisition traits that advantage one species over another, regardless of their frequency), which serve to strengthen interspecific competition (Chesson 2000). The limiting similarity hypothesis was developed at a time when ecologists were only aware of niche differences and the role these play in coexistence. Therefore, it may not be a surprise that ecologists have assumed niche differences grow larger, and interactions smaller, as closely related species evolve. But now that we know competition and coexistence depend on more than just niche differences (NDs), the limiting similarity hypothesis may need to be modified to account for other factors that influence the magnitude of competitive differences among species (RFDs). Traits representing species' niche differences (e.g., differential resource acquisition) and relative fitness differences (e.g., population growth rates) are both subject to the variety of selection mechanisms considered in the previous paragraph, and thus, may be distributed in any manner of contrasting ways across a phylogeny. In the strict case that both types of traits exhibit phylogenetic conservatism, increasing fitness differences along a phylogenetic gradient could counteract the competition-weakening effects of increasing niche differences along the same gradient, nullifying an overall evolutionary signal (Mayfield and Levine 2010). Teasing apart the relative importance of niche vs. fitness differences for species coexistence is a new goal for ecologists (Adler et al. 2007, Carroll et al. 2011, Hille Ris Lambers et al. 2012). But examining how both forces that influence coexistence influence phylogenetic limiting similarity is only just beginning (Narwani et al. 2013).

\section{Limits to inference: temporal, spatial, and phylogenetic scales}

While microcosm experiments using model biological systems are useful in testing mechanistic hypotheses that could not be accomplished in more natural systems, such studies obviously do not capture the full diversity and dynamics of natural communities. It should go without saying that our conclusions do not yet extend beyond the laboratory to natural communities of real lake phytoplankton, and conclusions from this study should remain tentative until further verification is performed with higher levels of reality. We would also caution that all inferences about phylogenies are contingent on the phylogenetic scale used to assess limiting similarity. For example, Peay et al. (2012) demonstrated scale-dependent changes in the relationship between phylogenetic distance and ecological similarity (although these changes did not affect the relationship between phylogenetic distance and species' interactions). We accounted for the potential scale dependency of our results by including a rich species pool distributed across a large phylogenetic gradient and testing the relationship between competitive strength and evolutionary history at multiple phylogenetic scales (i.e., within species, across all species, and across all species without two evolutionarily distinct taxa). Even so, we most certainly have not captured the full phylogenetic breadth of the species pools of North American phytoplankton, and thus, it is possible we failed to detect a signal of limiting similarity that might operate at even larger phylogenetic scales (e.g., the division between green algae vs. diatoms).

Most natural communities also contain multiple interacting species in a spatially heterogeneous and temporally fluctuating environment. We purposefully minimized such heterogeneity in our experiments so that we could explicitly minimize confounding effects that might limit our ability to measure the interspecific interaction strength of two competitors as a function of phylogenetic distance. In doing so, we have forced coexistence (or lack of) to be driven by sequestration of inorganic resources (e.g., nutrients and light) in a homogeneous environment. Our experiment certainly captured a broad range of interactions (from total competitive displacement, to coexistence, to facilitation), however, in natural communities more diverse resource environments (light and nutrient stratification through a larger water column, for example), multiple species interactions, and temporal variability in environmental conditions and biotic interactions may promote greater niche partitioning (and thus, reduced competitive interactions) than is possible in $1-\mathrm{mL}$ microcosm wells. 
With these caveats in mind, and with the recognition that we cannot say whether evolutionary relatedness influences species interactions under all scenarios faced in natural lakes, it is still striking that we could find no evidence to support the widespread assumption of phylogenetic limiting similarity across hundreds of combinations of interacting species using the same suite of inorganic resources. Our results support the growing understanding that phylogenetic limiting similarity may not be a ubiquitous phenomenon across ecological systems. Given the mixed results of the few explicit tests of this hypothesis, perhaps phylogenetic limiting similarity itself should be validated before the concept is used to explain patterns of structure and function in biological communities.

\section{ACKNOWLEDGMENTS}

We thank Patrick Venail and Anita Narwani for methodological and conceptual assistance in the formulation of this project. This work is support by the U.S. National Science Foundation's Dimensions of Biodiversity program in a grant to B. J. Cardinale and T. H. Oakley (DEB-1046121 and DEB1046307).

\section{Literature Cited}

Adler, P. B., J. Hille Ris Lambers, and J. M. Levine. 2007. A niche for neutrality. Ecology Letters 10:95-104.

Austin, M. P., L. F. M. Fresco, A. O. Nicholls, R. H. Groves, and P. E. Kaye. 1988. Competition and relative yield estimation and interpretation at different densities and under various nutrient concentrations. Journal of Ecology 76:157171.

Best, R. J., N. C. Caulk, and J. J. Stachowicz. 2013. Trait vs. phylogenetic diversity as predictors of competition and community composition in herbivorous marine amphipods. Ecology Letters 16:72-80.

Burns, J. H., and S. Y. Strauss. 2011. More closely related species are more ecologically similar in an experimental test. Proceedings of the National Academy of Sciences USA 108(13):5302-5307.

Cadotte, M. W., B. J. Cardinale, and T. H. Oakley. 2008. Evolutionary history and the effect of biodiversity on plant productivity. Proceedings of the National Academy of Sciences USA 105:17012-17017.

Cadotte, M. W., T. J. Davies, J. Regetz, S. Kembel, E. Cleland, and T. H. Oakley. 2010. Phylogenetic diversity metrics for ecological communities: integrating species richness, abundance and evolutionary history. Ecology Letters 13:96-105.

Cahill, J. F., Jr., S. W. Kembel, E. G. Lamb, and P. A. Keddy. 2008. Does phylogenetic relatedness influence the strength of competition among vascular plants? Perspectives in Plant Ecology, Evolution, and Systematics 10:41-50.

Carroll, I. T., B. J. Cardinale, and R. M. Nisbet. 2011. Niche and fitness differences relate the maintenance of diversity to ecosystem function. Ecology 92:1157-1165.

Catford, J. A., R. Jansson, and C. Nilsson. 2009. Reducing redundancy in invasion ecology by integrating hypotheses into a single theoretical framework. Diversity and Distributions 15:22-40.

Cavender-Bares, J., A. Keen, and B. Miles. 2006. Phylogenetic structure of Floridian plant communities depends on taxonomic and spatial scale. Ecology 87:S109-S122.

Cavender-Bares, J., K. Kozk, P. Fine, and S. Kembel. 2009. The merging of community ecology and phylogenetic biology. Ecology Letters 12:693-715.
Chesson, P. 2000. Mechanisms of maintenance of species diversity. Annual Review of Ecology and Systematics 31: 343-66.

Connolly, J., M. W. Cadotte, C. Brophy, A. Dooley, J. Finn, L. Kirwan, C. Roscher, and A. Weigelt. 2011. Phylogenetically diverse grasslands are associated with pairwise interspecific processes that increase biomass. Ecology 92:1385-1392.

Crisp, M. D., and L. G. Cook. 2012. Phylogenetic niche conservatism: what are the underlying evolutionary and ecological causes? New Phytologist 196:681-694.

Daehler, C. C. 2001. Darwin's naturalization hypothesis revisited. American Naturalist 158:324-330.

Darwin, C. 1859. The origin of species. John Murray, London, UK.

de Wit, C. T. 1960. On competition. Verslagen Van Landbouwkundige Onderzoekingen 66:1-82.

Dostál, P. 2011. Plant competitive interactions and invasiveness: searching for the effects of phylogenetic relatedness and origin on competition intensity. American Naturalist 177: 655-667.

Drummond, A. J., and A. Rambaut. 2007. BEAST: Bayesian evolutionary analysis by sampling trees. BMC Evolutionary Biology 7:214.

Edgar, R. C. 2004. MUSCLE: a multiple sequence alignment method with reduced time and space complexity. BMC Bioinformatics 5:1-19.

Faith, D. P. 1992. Conservation evaluation and phylogenetic diversity. Biological Conservation 61:1-10.

Flynn, D. F. B., N. Mirotchnick, M. Jain, M. I. Palmer, and S. Naeem. 2011. Functional and phylogenetic diversity as predictors of biodiversity-ecosystem-function relationships. Ecology 92:1573-1581.

Harper, J. L. 1977. Population biology of plants. Academic Press, London, UK.

Harvey, P. H., and M. D. Pagel. 1991. The comparative method in evolutionary biology. Oxford University Press, Oxford, UK.

Helmus, M. R., T. J. Bland, C. K. Williams, and A. R. Ives. 2007. Phylogenetic measures of biodiversity. American Naturalist 169:E68-E83.

Hille Ris Lambers, J., P. B. Adler, W. S. Harpole, J. Levine, and M. Mayfield. 2012. Rethinking community assembly through the lens of coexistence theory. Annual Review of Ecology, Evolution, and Systematics 43:227-248.

Ives, A. R., B. Dennis, K. L. Cottingham, and S. R. Carpenter. 2003. Estimating community stability and ecological interactions from time-series data. Ecological Monographs 73: 301-330.

Ives, A. R., and M. R. Helmus. 2010. Phylogenetic metrics of community similarity. American Naturalist 176:E128-E142.

Jiang, L., J. Tan, and Z. Pu. 2010. An experimental test of Darwin's naturalization hypothesis. American Naturalist 175:415-423.

Jolliffe, P. A. 2000. The replacement series. Journal of Ecology 88:371-385.

Kilham, S. S., D. A. Kreeger, S. G. Lynn, C. E. Goulden, and L. Herrera. 1998. COMBO: a defined freshwater culture medium for algae and zooplankton. Hydrobiologia 377:147159.

Kunstler, G., S. Lavergne, B. Courbaud, W. Thuiller, G. Vieilledent, N. E. Zimmermann, J. Kattge, and D. A. Coomes. 2012. Competitive interactions between forest trees are driven by species' trait hierarchy, not phylogenetic or functional similarity: implications for forest community assembly. Ecology Letters 15:831-840.

Laska, M. S., and J. T. Wootton. 1998. Theoretical concepts and empirical approaches to measuring interaction strength. Ecology 79:461-476.

Litchman, E., and C. A. Klausmeier. 2008. Trait-based community ecology of phytoplankton. Annual Review of Ecology, Evolution, and Systematics 39:615-639. 
Loreau, M., and A. Hector. 2001. Partitioning selection and complementarity in biodiversity experiments. Nature 412:7276.

Losos, J. B. 2008. Phylogenetic niche conservatism, phylogenetic signal and the relationship between phylogenetic relatedness and ecological similarity among species. Ecology Letters 11:995-1007.

MacArthur, R., and R. Levins. 1967. The limiting similarity, convergence, and divergence of coexisting species. American Naturalist 101:377-385.

Mayfield, M. M., and J. M. Levine. 2010. Opposing effects of competitive exclusion on the phylogenetic structure of communities. Ecology Letters 13:1085-1093.

Narwani, A., M. A. Alexandrou, T. H. Oakley, I. T. Carroll, and B. J. Cardinale. 2013. Experimental evidence that evolutionary relatedness does not affect the ecological mechanisms of coexistence in freshwater green algae. Ecology Letters 16:1373-1381.

Pavoine, S., S. Ollier, and A. B. Dufour. 2005. Is the originality of a species measurable? Ecology Letters 8:579-586.

Pearman, P. B., A. Guisan, O. Broennimann, and C. F. Randin. 2008. Niche dynamics in space and time. Trends in Ecology and Evolution 23:149-158.

Peay, K. G., M. Belisle, and T. Fukami. 2012. Phylogenetic relatedness predicts priority effects in nectar yeast communities. Proceedings of the Royal Society of London Series B 279:749-758.

Posada, D. 2008. jModelTest: Phylogenetic model averaging. Molecular Biology and Evolution 25:1253-1256.

Rodriguez-Ezpeleta, N., H. Brinkmann S. C. Burey, B. Roure, G. Burger, W. Loffelhardt, H. J. Bohnert, H. Phillipe, and B. F. Lang. 2005. Monophyly of primary photosynthetic eukaryotes: Green plants, red algae, and glaucophytes. Current Biology 15:1325-1330.

Snaydon, R. W. 1991. Replacement or additive designs for competition studies. Journal of Applied Ecology 28:930-946.

Srivastava, D. S., M. W. Cadotte, A. A. M. MacDonald, R. G. Marushia, and N. Mirotchnick. 2012. Phylogenetic diversity and the functioning of ecosystems. Ecology Letters 15:637648.
Stajich, J. E., et al. 2002. The bioperl toolkit: Perl modules for the life sciences. Genome Research 12:1611-1618.

Stamatakis, A., P. Hoover, and J. Rougemont. 2008. A rapid boostrap algorithm for the RAxML web servers. Systematic Biology 57:758-771.

Tan, J., Z. Pu, W. A. Ryberg, and L. Jiang. 2012. Species phylogenetic relatedness, priority effects, and ecosystem functioning. Ecology 93:1164-1172.

U.S. EPA [Environmental Protection Agency]. 2009. National lakes assessment: a collaborative survey of the nation's lakes. EPA 841-R-09-001. U.S. EPA, Office of Water and Office of Research and Development, Washington, D.C., USA.

Valiente-Banuet, A., and M. Verdú. 2007. Facilitation can increase the phylogenetic diversity of plant communities. Ecology Letters 10:1029-1036.

Vandermeer, J. 1989. The ecology of intercropping systems. Cambridge University Press, Cambridge, UK.

Verdú, M., L. Gomez-Aparicio, and A. Valiente-Banuet. 2012. Phylogenetic relatedness as a tool in restoration ecology: a meta-analysis. Proceedings of the Royal Society of London Series B 279:1761-1767.

Violle, C., D. R. Nemergut, Z. Pu, and L. Jiang. 2011. Phylogenetic limiting similarity and competitive exclusion. Ecology Letters 14:782-787.

Webb, C. O., D. D. Ackerly, M. A. McPeek, and M. J. Donoghue. 2002. Phylogenies and community ecology. Annual Review of Ecology and Systematics 33:475-505.

Weigelt, A., and P. Jolliffe. 2003. Indices of plant competition. Journal of Ecology 91(5):707-720.

Weiher, E., and P. A. Keddy. 1995. Assembly rules, null models, and trait dispersion: new questions from old patterns. Oikos 74:159-164.

Wiens, J. J., et al. 2010. Niche conservatism as an emerging principle in ecology and conservation biology. Ecology Letters 13:1310-1324.

Wiens, J. J., and C. H. Graham. 2005. Niche conservatism: integrating evolution, ecology, and conservation biology. Annual Review of Ecology, Evolution, and Systematics 36: 519-539.

\section{Supplemental Material}

\section{Appendix A}

Unsmoothed maximum likelihood phylogeny including 37 common North American freshwater green algae genera and three outgroups estimated using partial 18S ribosomal RNA and $\mathrm{rbcl}$ sequences available on GenBank (Ecological Archives E095-121-A1).

\section{Appendix B}

Identity, culture source, and North American distribution of green algae species used in this experiment (Ecological Archives E095-121-A2).

\section{Appendix C}

Illustration of experimental design highlighting individual plate inoculation, randomization across shaker platforms, and a photograph of the experimental chamber (Ecological Archives E095-121-A3).

\section{Appendix D}

Fluorescence time series data of each species' growth dynamics in monoculture (Ecological Archives E095-121-A4).

\section{Appendix E}

Logistic regression analysis illustrating the impact of evolutionary relatedness on the likelihood of coexistence (Ecological Archives E095-121-A5). 